\title{
Self-administered hyaluronidase facilitated subcutaneous immunoglobulin therapy in complicated primary antibody deficiencies
}

\begin{abstract}
Hyaluronidase facilitated subcutaneous immunoglobulin ( $\mathrm{fSClg}$ ) is a new immunoglobulin product for replacement therapy in patients with primary antibody deficiencies (PAD). The pre-administration of recombinant human hyaluronidase associated with $10 \%$ immunoglobulin allowed the infusion of larger (up to $600 \mathrm{ml}$ ) amounts of immunoglobulin at a single infusion site, enabling patients to receive the necessary treatment in a single monthly dose. Here, we report the effectiveness and the tolerability of $\mathrm{fSClg}$ in patients with severe PAD-related comorbidities: refractory autoimmune thrombocytopenia; systemic granulomatous disease; severe enteropathy, and Type I diabetes. We conclude that fSClg could be a feasible option to improve the adherence to replacement therapy also by patients with severe PAD.
\end{abstract}

First draft submitted: 15 March 2016; Accepted for publication: 10 June 2016; Published online: TBC

Keywords: common variable immunodeficiency $\bullet$ cytopenias $\bullet$ enteropathy $\bullet$ facilitated subcutaneous immunoglobulin $\bullet$ granulomatous disease $\bullet$ immunomodulation, intravenous immunoglobulin • subcutaneous immunoglobulin • thrombocytopenia • X-linked agammaglobulinemia

Primary antibody deficiencies (PAD) are characterized by a quantitative and/or qualitative impairment of antibody production. Common variable immunodeficiency (CVID) and X-linked agammaglobulinemia (XLA) are the most common symptomatic forms of PAD [1]. CVID includes a heterogeneous group of antibody deficiencies of unknown etiology, frequently diagnosed in adults, in which specific criteria could help in differentiating them from other inborn error of immunity. Across the spectrum of clinical manifestations, patients are frequently affected by severe and recurrent infections, autoimmune disorders, granulomatous and inflammatory diseases and cancers [2]. XLA is inherited as an X-linked recessive trait caused by Bruton's tyrosine kinase or BTK mutations resulting in a defective bone marrow $\mathrm{B}$ cell differentiation [3]. Improvements in awareness, prompt diagnosis and the introduction of immunoglobulin replacement therapy have resulted in substantially extended life expectancy for patients with PAD [4-6]. Treatment of PAD patients requires life-long replacement with human polyvalent immunoglobulins (Ig) administered by intravenous (IVIg), or subcutaneous (SCIg) routes [7,8]. A third route is intramuscular, although this is uncommonly. Recently, hyaluronidasefacilitated subcutaneous immunoglobulins (fSCIg) with recombinant human hyaluronidase (rHuPH20) pre-administered subcutaneously, followed by human immunoglobulin $10 \%$ infused through the same needle, has become available [9].

The pre-administration of $\mathrm{rHuPH} 20$ increases subcutaneous tissue permeability and facilitates drug dispersion and absorption, enabling the monthly administration of large amounts of product in one site with consequent increased intervals between
Maria Giovanna Danieli, ${ }^{* 1}$, Federica Pulvirenti², Valeria Rocchi ${ }^{3}$, Ramona Morariu ${ }^{1}$ \& Isabella Quinti ${ }^{2}$

${ }^{1}$ Clinica Medica, Dipartimento di Scienze Cliniche e Molecolari, Università Politecnica delle Marche \& Ospedali Riuniti, Ancona, Italy ${ }^{2}$ Department of Molecular Medicine, Sapienza Medical University, Rome, Italy ${ }^{3}$ Department of Clinical \& Sperimental Medicine AOUP, Pisa, Italy *Author for correspondence: m.g.danieli@univpm.it 
administrations compared with conventional SCIg [10]. fSCIg are dispensed after four steps of administration with increased dosages injected at short interval times called ramp-up schedule. This schedule permits patients to become adapted to a large volumes of liquid administered subcutaneously. After the initial rampup, fSCIg are administered at 300 to $600 \mathrm{mg} / \mathrm{kg}$ at 3-4 week intervals.

Data have already shown a good efficacy profile and a low rate of adverse reactions to fSCIg allowing home-based administration for adequately trained patients [11,12]. However, few data are available on the efficacy and tolerability of fSCIg in patients with PAD-associated complicated clinical conditions such as enteropathy, cytopenias and granulomatous disease. These comorbidities require a careful personalized evaluation of immunoglobulin dosages and schedules of administration in order to improve the clinical outcome [13]. Here, we report on five patients diagnosed according to the ESID/PAGID criteria, affected by serious PAD-related comorbidities. Clinical, immunological data, reason to start fSCIg therapy and data after the treatment were summarized in Table 1.

\section{Case 1}

A 58-year-old woman came to our attention after a long-standing history of upper respiratory tract infections and refractory idiopathic thrombocytopenia (ITP) treated with glucocorticoids and splenectomy. CVID was diagnosed based on low serum immunoglobulin levels (IgG $82 \mathrm{mg} / \mathrm{dl}, \operatorname{IgA} 25 \mathrm{mg} / \mathrm{dl}$, IgM 47 $\mathrm{mg} / \mathrm{dl}$ ) and impaired specific antibody responses, after exclusion of secondary hypogammaglobulinemias. The patient began IVIg at the dosage of $0.6 \mathrm{~g} / \mathrm{kg} / \mathrm{month}$. Platelet (PLT) count increased from $30,000 / \mathrm{mm}^{3}$ to $130,000 / \mathrm{mm}^{3}$. After 4 years, in December 2014, she presented with mild mucocutaneous haemorrhages associated with a PLT count of $53,000 / \mathrm{mm}^{3}$. Laboratory tests revealed normal red blood cell, reticulocyte and white blood cell counts and no abnormalities on peripheral blood smear. Anti-PLT autoantibodies were positive. Bone marrow biopsy showed an increase in megakaryocytes. Abdominal ultrasound scan demonstrated the presence of residual splenic tissue. Since she could not tolerated an increased IVIg dosage and she refused to receive two divided IVIg doses, she was switched to fSCIg. The product was administered by a programmable pump without petechial hemorrhaging at the infusion site. We used a standardized infusion protocol with a velocity/dose ramp-up (as shown in Figure 1) and we achieved the IVIg equivalent monthly dose after four infusions. The patient received fSCIg $20 \mathrm{~g}$ twice a month and IgG trough levels remained constantly above $750-850 \mathrm{mg} / \mathrm{dl}$. After two infusions, the PLT values rose from $53,000 / \mathrm{mm}^{3}$ to $122,000 /$ $\mathrm{mm}^{3}$ and subsequently remained $>80,000 / \mathrm{mm}^{3}$ for the following 7 months (Figure 1) without any further episode of mucocutaneous hemorrhage.

\section{Case 2}

A 60-year-old woman attended our clinic for recurrent infections and thrombocytopenia. On initial examination (December 2014) she had cutaneous and mucosal petechiae. Laboratory evaluation showed low PLT count $\left(58,000 / \mathrm{mm}^{3}\right)$, normal erythrocyte and leukocyte counts. Anti-PLT autoantibodies were undetectable.

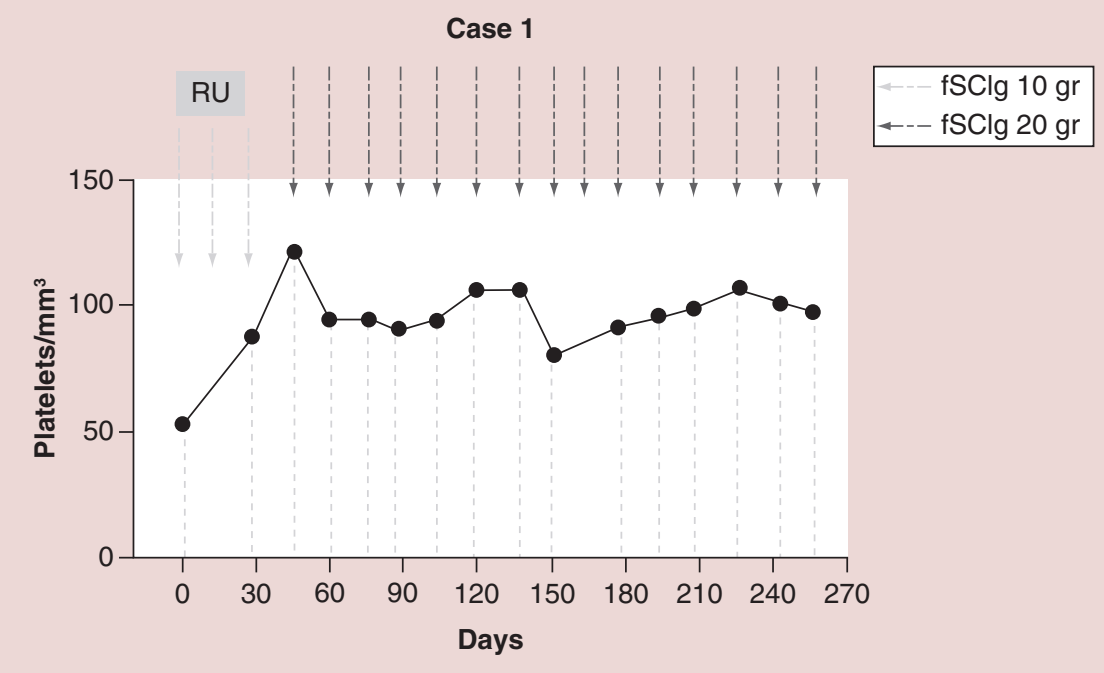

Figure 1. Platelet count in a common variable immunodeficiency patient affected by refractory thrombocytopenia. After the switch to $\mathrm{fSClg}$ therapy, patient had a persistent increase in platelet count. Patient started with a fSClg dose of $10 \mathrm{~g}$ followed a week later with a $20 \mathrm{~g}$ dose and 2 weeks later with a $30 \mathrm{~g}$ dose at 3-week interval. fSClg: Facilitated subcutaneous immunoglobulin. 


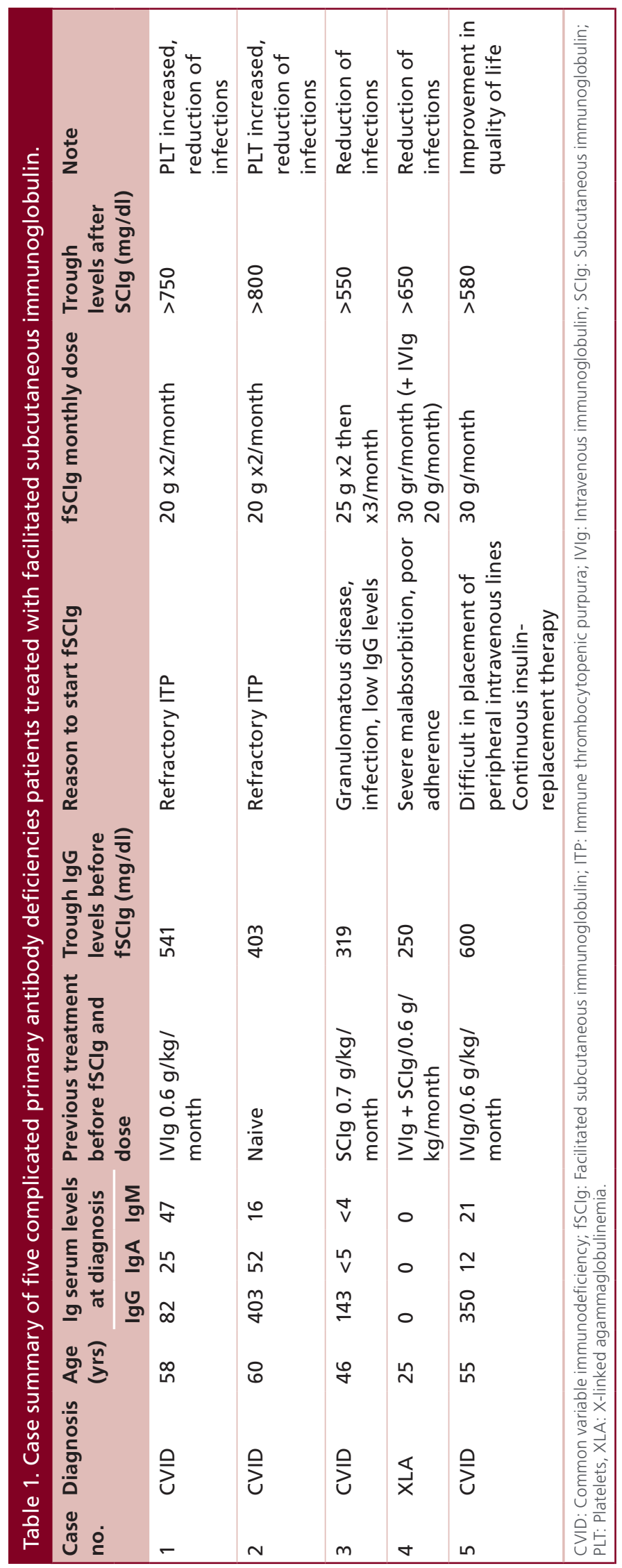

She had low serum Ig levels (IgG 403; IgA 52; IgM $16 \mathrm{mg} / \mathrm{dl}$ ) and impaired response to tetanus toxoid. After exclusion of secondary hypogammaglobulinemias, she was diagnosed with CVID. Due to the poor venous access, she started fSCIg as an immunoglobulin replacement treatment. Afterwards ramp-up schedule (as shown in Figure 2), $\mathrm{fSCIg}$ were administered at the dosage of $20 \mathrm{~g}$ biweekly achieving the dosage of $0.6 \mathrm{~g} / \mathrm{kg} /$ month. After the second infusion, PLT rose from $58,000 / \mathrm{mm}^{3}$ to $93,000 / \mathrm{mm}^{3}$ (Figure 2) with resolution of petechiae. Serum IgG levels reached a protective value after two infusions $(550 \mathrm{mg} / \mathrm{dl})$, remaining above $800 \mathrm{mg} / \mathrm{dl}$ in the subsequent year. No infectious episodes were documented. Platelet counts remained constantly $>100,000 /$ $\mathrm{mm}^{3}$. Mild or moderate redness occurred at the injection site but disappeared without sequelae by lowering the infusion rate to $140 \mathrm{ml} / \mathrm{h}$. No petechial hemorrhaging was recorded at the infusion site.

\section{Case 3}

A 46-year-old man was affected by CVID complicated by granulomatous systemic disease. He came to our attention at the age of 40 years because of a marked reduction of serum levels of $\operatorname{IgG}$ and $\operatorname{IgA}$ with a clinical history of recurrent infections. The CVID diagnosis was make at the age of 27 years, but the replacement therapy with IVIg was performed in an irregular manner in the reference hospital, then stopped for serious adverse reaction. After the first examination, in absence of replacement therapy for several months, he was hospitalized because he developed fever, lymph node enlargement, infiltrated skin lesions and episodes of abdominal pain. Skin biopsy showed granulomatous dermatitis. The computed tomography showed splenomegaly and enlarged superficial mediastinal and abdominal non-confluent lymph nodes. Lymph node and bone marrow biopsies revealed non-necrotizing epithelioid granulomas. In the following years, renal and hepatic biopsy were also performed due to a progressive renal function impairment and to increased liver enzyme serum levels. Liver and kidney histology showed a smallmedium size lymphocyte infiltrate. However, no conclusive evidence of a lymphoproliferative disease was obtained. Oral cyclophosphamide was also started and continued up to a total dose of $9 \mathrm{~g}$ followed by other immuno- 


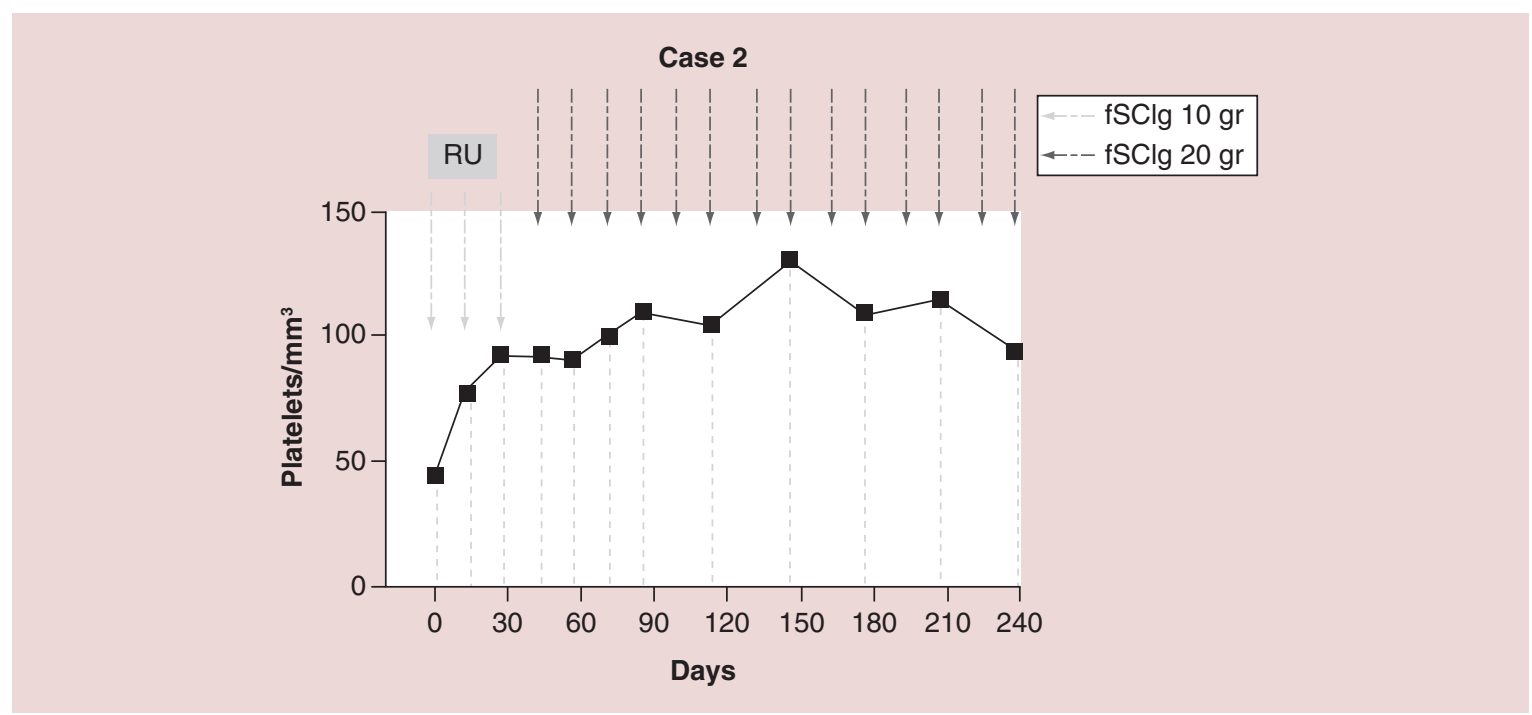

Figure 2. Platelet count in a naive common variable immunodeficiency patient affected by thrombocytopenia. Since the diagnosis the patient was treated with $\mathrm{fSClg}$. Platelet counts increased to $100,000 / \mathrm{mm}^{3}$ and serum IgG levels reached a protective value after two $\mathrm{fSClg}$ administrations. Platelet counts remained constantly $>100,000 /$ $\mathrm{mm}^{3}$ during the follow-up.

fSClg: Facilitated subcutaneous immunoglobulin.

suppressant drugs (mycophenolate mofetil at $30 \mathrm{mg} /$ $\mathrm{kg} /$ day orally). After 8 months, renal and liver function improved. Despite conventional SCIg infusion administered at $0.7 \mathrm{~g} / \mathrm{kg} / \mathrm{month}$, trough $\mathrm{IgG}$ levels progressively decreased to $200 \mathrm{mg} / \mathrm{dl}$ and the number of respiratory infections increased. Antibiotic prophylaxis was initiated At the end of 2014, fSCIg was available in Italy and the patient was switched to fSCIg with a ramp-up protocol. After ramp-up, satisfactory response was documented by the increase in serum IgG levels from 319 to $648 \mathrm{mg} / \mathrm{dl}$ (Figure 3) and by a reduction of infectious episodes. Initially, the patient received fSCIg $25 \mathrm{~g}$ twice a month, then the interval was increase to a 3-weekly administration with a consequent drop of IgG trough levels that remained constantly $>550 \mathrm{mg} / \mathrm{dl}$. After 12 months, the granulomatous disease caused a catastrophic multiorgan failure and the patient died.

\section{Case 4}

A 25-year-old man had a diagnosis of XLA complicated by severe malabsorption and weight loss. His clinical history was characterized by recurrent episodes of pneumonia and chronic diarrhea. Duodenal biopsy showed increased count of intraepithelial lymphocytes (>30/100 epithelial cells) associated with severe intestinal villous atrophy. Initially, a coeliac disease was suspected. The diagnosis of celiac disease is difficult in patients with PAD, due to the impossibility to use serological data. The presence of HLA DQ2 and DQ8 could be helpful [14]; however, the response to a gluten free diet remains the main diagnostic criteria [15].
The patient did not respond to a gluten-free diet so he was diagnosed as an duodenal villous atrophy associated to the immunodeficiency [16]. Replacement therapy was challenging in that IVIg administered at a cumulative dose of $40 \mathrm{~g} /$ month $(0.7 \mathrm{~g} / \mathrm{kg} /$ month $)$ did not achieve a satisfactory clinical and immunological response. We increased the Ig monthly cumulative dose up to $50 \mathrm{~g} / \mathrm{month}(0.8 \mathrm{~g} / \mathrm{kg} / \mathrm{month})$ by administering IVIg and SCIg at the same time. This allowed a good clinical response with IgG trough levels persistently $>600 \mathrm{mg} / \mathrm{dl}$ in the absence of major infections. However, the patient had poor compliance with SCIg administration due to social and family background disadvantage. He progressively self-reduced the cumulative Ig monthly dose to $30 \mathrm{~g}$ with a consequent drop in the IgG trough levels (Figure 4A). At that time he had an episode of pneumonia. In December 2014, to reduce the number of subcutaneous Ig administration, and possibly improve the adherence, he started fSCIg according to the ramp-up protocol. After $40 \mathrm{~g}$ of fSCIg his IgG trough levels remained very low $(200 \mathrm{mg} / \mathrm{dl})$ and we had to introduce a single IVIg administration soon after the ramp-up to achieve a total a cumulative monthly dose of $50 \mathrm{gr}$. This allowed for a satisfactory IgG trough level and administration of monthly fSCIg doses. Unfortunately, the patient was again not adherent to the IVIg therapy and, 20 days after the last fSCIg administration, the IgG trough level dropped again to $100 \mathrm{mg} / \mathrm{dl}$ and the patient had a new episode of pneumonia requiring hospitalization (Figure 4B). Later on, the patient shifted back to IVIG at a cumulative monthly dosage of $50 \mathrm{~g} / \mathrm{month}$. However, the 


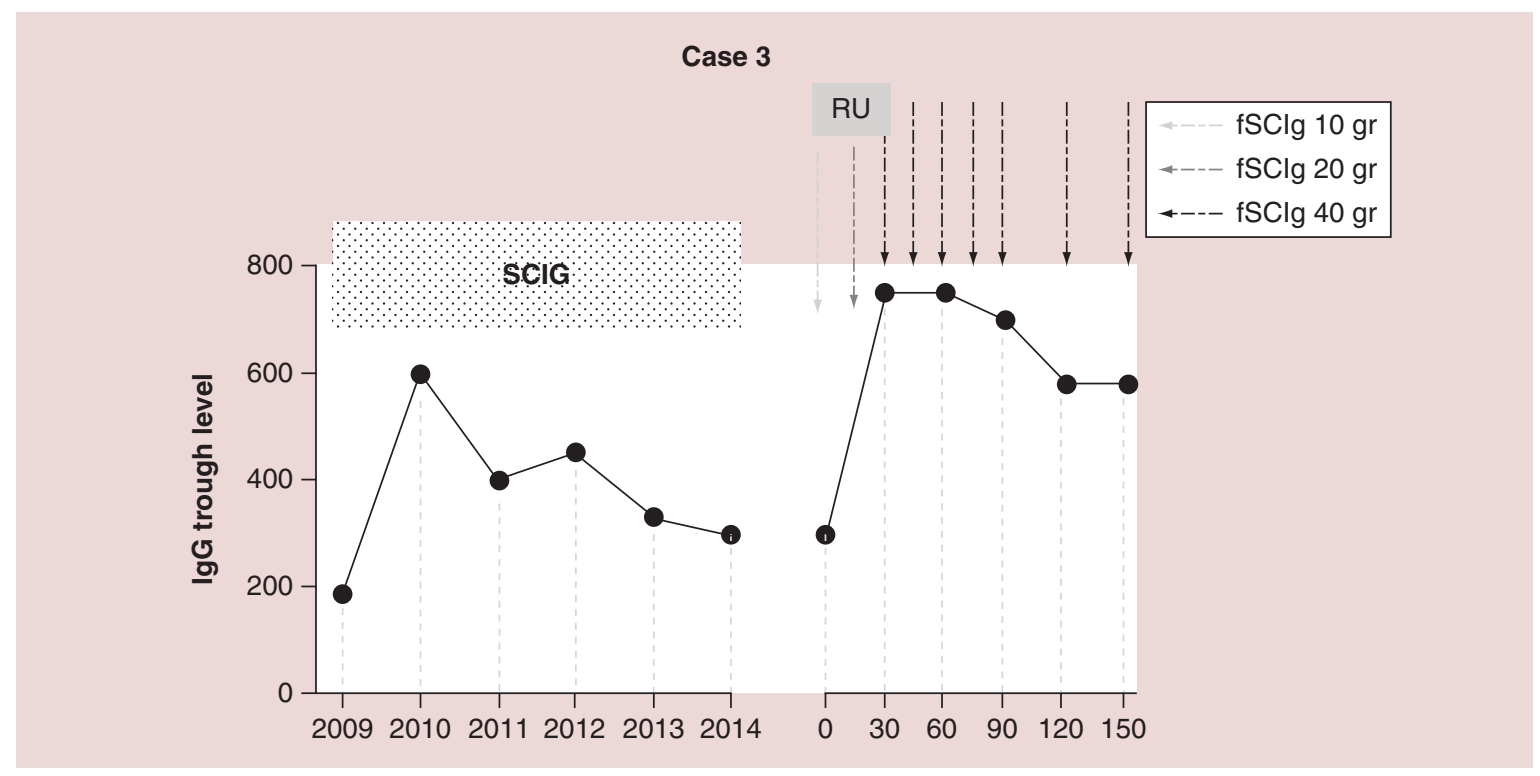

Figure 3. IgG serum levels in a common variable immunodeficiency patient suffering from systemic granulomatous disease and recurrent severe infections who previously failed several conventional SClg treatment schedules. In December 2014, the patient switched to fSClg and reached protective serum Ig levels. During the follow-up, the patient remained infection free. However, the granulomatous disease progressively led to a catastrophic multiorgan failure and the patient died.

fSClg: Facilitated subcutaneous immunoglobulin.

serum trough IgG levels remained below $4.0 \mathrm{~g} / \mathrm{l}$. The patient was encouraged to start again a combination of fSCIg $(30 \mathrm{~g} /$ month $)$ and IVIg $(20 \mathrm{~g} /$ month $)$, in order to improve the adherence and to achieve a satisfactory IgG trough levels ( $>650 \mathrm{mg} / \mathrm{dl})$.

\section{Case 5}

A 55-year-old man had a diagnosis of CVID with a clinical history of bronchiectasis and chronic sinusitis and a diagnosis of insulin dependent diabetes since infancy. Thus, he had to replace both immunoglobulin and insulin using a bimonthly IVIg administration and a continuous infusion of rapid-acting insulin by pump delivery. Replacement therapy with IVIg was efficacious and well tolerated at a monthly cumulative dosage of $30 \mathrm{~g}$. However, recently the placement of peripheral intravenous lines became extremely difficult. The patient refused conventional SCIg since he was on continuous insulin infusion. In January 2015 he switched to fSCIg; according to the ramp-up protocol, after three initial doses, he could reach the same dosage and frequency of IVIg treatment. This allowed a satisfactory IgG trough level 4 months after the initial dose at the time of the monthly fSCIg administration (Figure 5).

\section{Discussion}

Ig replacement is the standard therapy for PAD, which aims to replace the missing antibodies and to prevent recurrent infections. Replacement therapy generally encompasses the use of $400-600 \mathrm{mg} / \mathrm{kg}$ of human immunoglobulin administered intravenously every 3 or 4 weeks or subcutaneously daily to every 2 weeks. The optimal choice of timing and administration route is indeed an important determinant of the adherence to IgG long-term therapy and patient outcomes. Research into individualized medicine and personalized health presents methodological challenges. In PAD, different options have been explored to establish how we should individualize Ig replacement therapy. As with many interventions, there may be specific subgroups of patients who are more likely to benefit from treatment with higher or lower dosages of immunoglobulins. Moreover, there are PAD patients who did not achieve a satisfactory efficacy, despite immunoglobulin replacement administered according to the international guidelines [13]. Recently, the introduction of hyaluronidase fSCIg has allowed the infusion of larger (up to $600 \mathrm{ml}$ ) amounts of Ig at a single infusion site. This new product might enable the patient to receive the replacement treatment with a single monthly dose, with a possible improvement in adherence and quality of life [9,17-19]. Here, we reported on a series of PAD patients with serious comorbidities poorly responsive to conventional IVIg or SCIg who shifted to replacement with fSCIg. All remained free from severe infections with the exception of an XLA patient who suffered from critical malabsorption. Interesting, for this patient only a high IgG cumulative monthly dosage 
administered using a combination of fSCIg and IVIg resulted in a clinical and immunological improvement. Various hypotheses can be evoked to explain this effect of fSCIg replacement therapy in a patient with a severe protein losing enteropathy: first, the use of fSCIg (and IVIg) improved the patient's adherence; second, trough levels increased due to the new pharmacokinetics. In fact, fSCIg administered subcutaneously are slowly absorbed and redistributed, with a probably low dispersion. An improvement of IgG trough levels and a reduction of infectious episodes was also documented in the patient affected by a life-threatening critical granulomatous CVIDassociated disease. The treatment with fSCIg had a good tolerability. Our patients did not have systemic side effects or complain of any discomfort from treatment. Moreover, fSCIg therapy was well tolerated also in the patient with diabetes who was under an additional long-life continuous treatment with insulin and in the two patients with thrombocytopenia, who did not report any local hemorrhagic manifestations at the infusion site. In these two patients, fSCIg improved the refractory thrombocytopenia. According to Seidel [20], thrombocytopenia in PAD could be a primary feature of the immunodeficiency, being autoantibody-mediated and/or cell-mediated. At this regard, we speculate about the possible immunomodulatory role of fSCIg which can reflect that of IVIg, even if fSCIg has a slightly lower serum Ig level peak as compared with IVIg. In immune-mediated

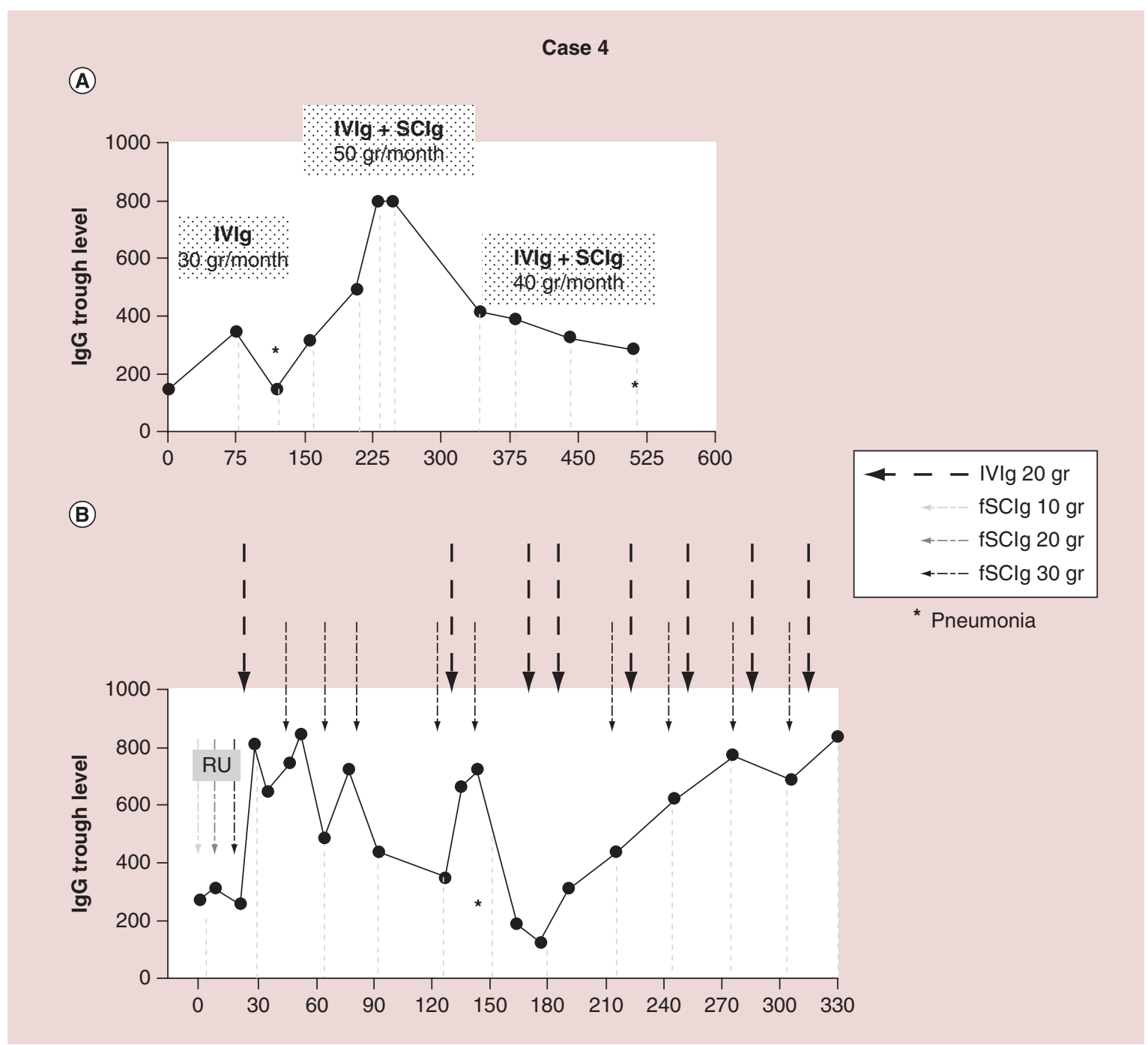

Figure 4. IgG serum levels in a X-Linked agammaglobulinemia patient affected by duodenal villous atrophy during IVIg and conventional SClg treatment (A) and IVIg plus fSClg treatment (B). Before 2014, the patient had several pneumonia episodes requiring hospitalization. He reached protective IgG levels only by a combination of IVIg and SClg, with a cumulative monthly dose of $50 \mathrm{~g} / \mathrm{month}$. Because of his low adherence to therapy, the patient switched to a combination of IVIg and fSClg at the same monthly dosage.

fSClg: Facilitated subcutaneous immunoglobulin.; IVIg: Intravenous immunoglobulin. 


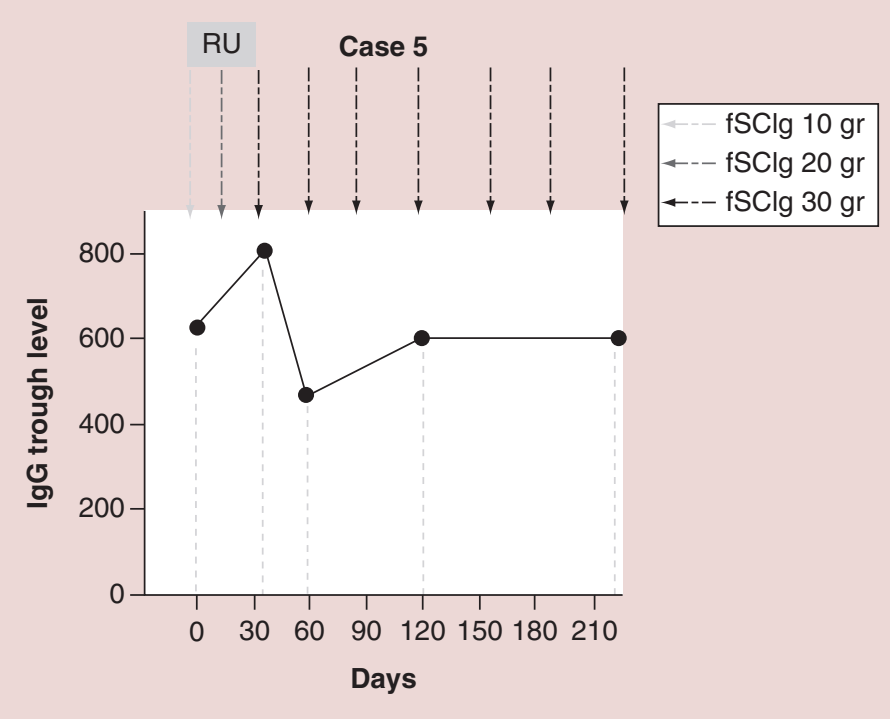

Figure 5. IgG serum levels in a common variable immunodeficiency patient affected by insulin-dependent diabetes on continuous insulin-replacement therapy.

During the follow-up patient had a good adherence to therapy and maintained protective IgG serum levels. fSClg: Facilitated subcutaneous immunoglobulin.

diseases, several mechanisms of action have been suggested to explain the molecular and cellular pathways that are involved in IVIg-mediated immunomodulation, depending on the nature of the disease [21-25]. It is also probable that more than one mechanism is responsible for the beneficial effect of IVIg in certain diseases. Recently conventional SCIg have been successfully employed in immune-mediated diseases such as chronic inflammatory demyelinating neuropathy, multifocal motor neuropathy and idiopathic inflammatory myopathies [26]. The immunodulatory mechanism of action of SCIg (and of facilitated SCIg) is not completely understood. We can speculate that an alternative molecular target to IVIg may be involved due to the subcutaneous route of administration.

Although more data and a longer follow-up period are necessary to verify the effectiveness of fSCIg in PAD patients affected by serious comorbidities, our preliminary findings document a good tolerance of fSCIg. For these patients, fSCIg could be a feasible option to improve adherence to therapy and possibly improve PAD-related comorbidities and quality of life.

\section{Financial \& competing interests disclosure}

MG Danieli, V Rocchi and I Quinti have received support from Baxalta and CSL Behring, for conferences and research. The authors have no other relevant affiliations or financial involvement with any organization or entity with a financial interest in or financial conflict with the subject matter or materials discussed in the manuscript apart from those disclosed.

No writing assistance was utilized in the production of this manuscript.

\section{Ethical conduct of research}

The authors state that they have obtained appropriate institutional review board approval or have followed the principles outlined in the Declaration of Helsinki for all human or animal experimental investigations. In addition, for investigations involving human subjects, informed consent has been obtained from the participants involved.

\section{Informed consent disclosure}

The authors state that they have obtained verbal and written informed consent from the patient/patients for the inclusion of their medical and treatment history within this case report.

\section{Executive Summary}

- The administration of facilitated subcutaneous immunoglobulin ( $\mathrm{fSClg}$ ) was effective in maintaining adequate serum IgG levels and in reducing the infections in a series of five patients with common variable immunodeficiency.

- fSClg might have an immunomodulatory potential as seen in two patients who improved their common variable immunodeficiency associated immune-mediated thrombocytopenia.

- The administration of $\mathrm{fSClg}$ was well tolerated and patients reported no relevant side effects. 


\section{References}

Papers of special note have been highlighted as:

- of interest; $\bullet$ of considerable interest.

1 Notarangelo LD. Primary immunodeficiencies. J. Allergy. Clin. Immunol. 125(2), S182-S194 (2010).

2 Chapel H, Lucas M, Lee M et al. Common variable immunodeficiency disorders: division into distinct clinical phenotypes. Blood 112(2), 277-286 (2008).

3 Winkelstein JA, Marino MC, Lederman HM et al. X-linked agammaglobulinemia: report on a United States registry of 201 patients. Medicine (Baltimore) 85(4), 193-202 (2006).

4 Busse PJ, Razvi S, Cunningham-Rundles C. Efficacy of intravenous immunoglobulin in the prevention of pneumonia in patients with common variable immunodeficiency. $J$. Allergy Clin. Immunol. 109(6), 1001-1004 (2002).

5 Orange JS, Hossny EM, Weiler CR et al. Use of intravenous immunoglobulin in human disease: a review of evidence by members of the Primary Immunodeficiency Committee of the American Academy of Allergy, Asthma and Immunology. J. Allergy Clin. Immunol. 117(6), S525-S553 (2006).

6 Quinti I, Soresina A, Guerra A et al. Effectiveness of immunoglobulin replacement therapy on clinical outcome in patients with primary antibody deficiencies: results from a multicenter prospective cohort study. J. Clin. Immunol. 31(3), 315-322 (2011).

7 Huang F, Feuille E, Cunningham-Rundles C. Home care use of intravenous and subcutaneous immunoglobulin for primary immunodeficiency in the United States. J. Clin. Immunol. 33(1), 49-54 (2013).

8 Berger M. Choices in IgG replacement therapy for primary immune deficiency diseases: subcutaneous IgG vs. intravenous IgG and selecting an optimal dose. Curr. Opin. Allergy Clin. Immunol. 11(6), 532-538 (2011).

9 Wasserman RL, Melamed I, Stein MR et al. IGSC, 10\% with rHuPH20 Study Group. Recombinant human hyaluronidase-facilitated subcutaneous infusion of human immunoglobulins for primary immunodeficiency. J. Allergy Clin. Immunol. 130(4), 951-957 (2012).

10 Kang DW, Jadin L, Nekoroski T, Drake FH, Zepeda ML. Recombinant human hyaluronidase PH20 (rHuPH20) facilitates subcutaneous infusions of large volumes of immunoglobulin in a swine model. Drug Deliv. Transl. Res. 2(4), 254-264 (2012).

11 Sanford M. Human immunoglobulin 10\% with recombinant human hyaluronidase: replacement therapy in patients with primary immunodeficiency disorders. BioDrugs 28(4), 411-420 (2014).

12 Wasserman RL. Overview of recombinant human hyaluronidase-facilitated subcutaneous infusion of $\mathrm{IgG}$ in primary immunodeficiencies. Immunotherapy 6(5), 553-567 (2014).

- A clear and concise review on facilitated subcutaneous immunoglobulin from the pharmacokinetics data to first clinical results in patients with primary immunodeficiency disorders (PID).

13 Milito C, Pulvirenti F, Pesce AM et al. Adequate patient's outcome achieved with short immunoglobulin replacement intervals in severe antibody deficiencies. J. Clin. Immunol. 34(7), 813-819 (2014).

-. Gives important issues on how to fix the exact timing and the optimal immunoglobulin replacement treatment modalities consistent with clinical and immunological features.

14 Venhoff N, Emmerich F, Neagu M et al. The role of HLA DQ2 and DQ8 in dissecting celiac-like disease in common variable immunodeficiency. J. Clin. Immunol. 33(5), 909-916 (2013).

15 Biagi F, Bianchi PI, Zilli A et al. The significance of duodenal mucosal atrophy in patients with common variable immunodeficiency: a clinical and histopathologic study. Am. J. Clin. Pathol. 138(2), 185-189 (2012).

16 Malamut G, Verkarre V, Suarez F et al. The enteropathy associated with common variable immunodeficiency: the delineated frontiers with celiac disease. Am. J. Gatroenterol. 105(10), 2262-2275 (2010).

17 Jolles S. Hyaluronidase facilitated subcutaneous immunoglobulin in primary immunodeficiency. ImmunoTargets Ther. 2, 125-133 (2013).

18 Ponsford M, Carne E, Kingdon C et al. Facilitated subcutaneous immunoglobulin (fSCIg) therapy - practical considerations. Clin. Exp. Immunol. 182(3), 302-313 (2015).

19 Carne E, Ponsford M, El-Shanawany T, Williams P, Pickersgill T, Jolles S. Five years of self-administered hyaluronidase facilitated subcutaneous immunoglobulin (fSCIg) home therapy in a patient with primary immunodeficiency. J. Clin. Pathol. 69(1), 87-88 (2016).

20 Seidel MG. Autoimmune and other cytopenias in primary immunodeficiencies: pathomechanisms, novel differential diagnoses, and treatment. Blood 124(15), 2337-2344 (2014).

21 Imbach P. 30 years of immunomodulation by intravenous immunoglobulin. Immunotherapy 4(7), 651-654 (2012).

22 Kaveri SV, Lecerf M, Saha C et al. Intravenous immunoglobulin and immune response. Clin. Exp. Immunol. 178(Suppl. 1), 94-96 (2014).

23 Kaveri SV, Maddur MS, Hedge P, Lacroix-Desmazes S, Bayry J. Intravenous immunoglobulins in immunodeficiencies: more than mere replacement therapy. Clin. Exp. Immunol. 164(Suppl. 2), 2-5 (2011).

-. Reviews the rationale for the intravenous immunoglobulin in immunodeficiencies, underlying its immunomodulatory role.

24 Maddur MS, Othy S, Hegde P et al. Immunomodulation by intravenous immunoglobulin: role of regulatory $\mathrm{T}$ cells. $J$. Clin. Immunol. 30 (Suppl. 1), S4-S8 (2011).

25 Kessel A, Ammuri H, Peri R et al. Intravenous immunoglobulin therapy affects $\mathrm{T}$ regulatory cells by increasing their suppressive function. J. Immunol. 179(8), 5571-5575 (2007)

26 Danieli MG, Gelardi C, Pedini V et al. Subcutaneous IgG in immune-mediate diseases: proposed mechanisms of action and literature review. Autoimmun. Rev. 13(12), 1182-1188 (2014). 